\title{
Observational evidences for the speed of the gravity based on the Earth tide
}

\author{
TANG KeYun ${ }^{1,2 *}$, HUA ChangCai ${ }^{3}$, WEN Wu ${ }^{1}$, CHI ShunLiang ${ }^{4}$, YOU QingYu ${ }^{1} \&$ YU Dan ${ }^{5}$ \\ ${ }^{1}$ Institute of Geology and Geophysics, Chinese Academy of Sciences, Beijing 100029, China; \\ ${ }^{2}$ National Astronomical Observatory, Chinese Academy of Sciences, Beijing 100012, China; \\ ${ }^{3}$ Institute of Geophysics, China Earthquake Administration, Beijing 100081, China; \\ ${ }^{4}$ University of Chinese Academy of Sciences, Beijing 100049, China; \\ ${ }^{5}$ Network Center of China Earthquake Administration, Beijing 100045, China
}

Received September 17, 2012; accepted October 16, 2012; published online November 28, 2012

\begin{abstract}
We have found that the current practical Newtonian formula for the gravity tide of the Earth implies a hypothesis that gravity travels at the speed of light; furthermore, we have derived and solved the propagation equation of gravity using the observation data of Earth tides from Shiquanhe and Wushi, after correction of phase lag due to the anelasticity of the Earth, and found that the speeds of gravity are from 0.93 to 1.05 times the speed of light with a relative error of about $5 \%$. This provides first set of strong evidences to show that the speed of gravity is the same as the speed of light.
\end{abstract}

Earth tide, apparent position, phase lag, speed of light, speed of gravity, propagation equation of gravity

Citation: Tang K Y, Hua C C, Wen W, et al. Observational evidences for the speed of the gravity based on the Earth tide. Chin Sci Bull, 2013, 58: 474-477, doi: $10.1007 / \mathrm{s} 11434-012-5603-3$

\section{Introduction of gravitation and Earth tide}

Although the theory of gravity was proposed much earlier than the theory of electromagnetism, the latter is much more clearly understood by the scientific community whereas many questions remain on the nature of the former. It is well known that Newton's law of gravitation is a theory of action at a distance, i.e. gravity can be instantaneously transmitted to any place, or the speed of gravity is infinite. However, Einstein's special relativity theory states that the speed of light is the upper limit for all physical speeds; therefore the speed of the gravity should be finite. Furthermore general relativity assumes that disturbing gravity such as gravitational waves should travel at the speed of light [1-4]. These theories are clearly at odds with each other. To resolve such a significant theoretical puzzle, we need sound

*Corresponding author (email: kytang@bao.ac.cn) experimental or observational results rather than pure logical debate. Since gravity cannot be shielded, and because our Earth is relatively small, it is nearly impossible to measure the speed of gravity inside a laboratory. So far, physicists have not found any widely recognized method to measure the propagation speed of gravity, nor measured the speed of gravity.

Since March of 1997, we had carried out 6 observations of the total or annular solar eclipse, including observations of the gravity tide of the Earth. The Earth tides are essentially the motions in the solid earth induced by the lunar and solar tidal forces. These tidal forces act on the anelastic solid Earth and cause distortions of Earth or the mass redistribution of Earth; this mass redistribution produces an additional force. The vector sum of the lunar tidal force, solar tidal force and this additional force creates the phase lag of Earth tide, i.e. the phase of the observational curves lags that of the theoretical curve of Earth tide [5,6].

Practical Newtonian formula of solar tidal force. Based 
on the classical Newtonian gravitation, geophysicists and astrophysicists construct the Earth tide model. For example, the solar tidal field is

$$
\vec{S}(O, t)=-\frac{G M_{s}}{r_{*}^{3}\left(t_{*}\right)} \vec{r}_{*}\left(t_{*}\right)+\frac{G M_{s}}{R_{*}^{3}\left(t_{*}\right)} \vec{R}_{*}\left(t_{*}\right),
$$

where $\vec{r}$ and $\vec{R}$ are the position vectors from the center of the Sun to the observing station and the center of Earth respectively; the first term on the right side of eq. (1) is the difference between the two terms, the first rerm is the Newtonian gravitational field of the Sun acting on the observation point, and the second term is the Newtonian gravitational field of the Sun acting on the center of the Earth. So the solar tidal field is equivalent to the acceleration of the test mass at the observation point relative to the center of the Earth under the gravitational action of the Sun. In eq. (1), all positions with subscript ' $*$ ' indicate that the positions are all the apparent positions. Since the true positions of the Sun cannot be observed directly, geophysicists and astrophysicists have to use the apparent positions of the Sun instead of corresponding true positions in the Newtonian formula of solar tidal force $[5,6]$, i.e. the solar tidal force that gets calculated is actually the tidal force of the Sun as it was issued from its apparent position and at a retarded time $t_{*}=t-\Delta t\left(\Delta t \equiv \frac{r_{*}}{c}\right)$ rather than the observation time $t[7,8]$.

Hence we realized that the practical Newtonian formula of solar tidal force has included the influence of the propagation of the gravity, and then proposed the following proposition: the current practical Newtonian formula of gravity tide of the Earth actually implies a hypothesis that gravity travels at the speed of light [9-11].

Eq. (1) shows us that the time of gravity issued from the Sun, and the time of gravity received at ground station are not the same! This is contradictory to the classical Newtonian gravitational law which states the time of gravity issued from the source of gravity and the time of gravity received at observation point are the same. The time elapsed corresponds exactly to the time interval during which light travels from the Sun to the ground observation point. This is also correct for the lunar tidal formula. So, the current practical tidal formula actually implies a hypothesis that gravity travels at the speed of light.

Based on the practical Newtonian formula of solar tidal force, if we add the time interval $\Delta t$ defined above to the retarded time $t_{*}$ for calculating the apparent position, we arrive at the true position of the Sun. In other words, this is equivalent to constructing a real instantaneous propagation formula for the solar tidal force.

\section{The propagation equation of gravity of the Sun}

For the solar tidal force, let us consider the difference of arrival times between the observation curve and the practical Newtonian formula at each curve's zero-tide points. The time difference has two origins: the first is the phase lag due to the delayed response of anelastic Earth to solar tidal force, and the second is the time difference caused by the travel speed from the Sun to the ground station, if the real travel speed $u$ is different from the speed of light $c$. Then we arrive at the propagation equation of gravity [10,11], after separating the solar tide from the observation of the Earth tide. The propagation equation of gravity of the Sun should be

$$
t_{\text {corr }}^{\text {obse }}-t_{\text {prac }}^{\text {theo }}=\frac{r_{*}}{u}-\frac{r_{*}}{c} .
$$

On the left side of the eq. (2), $t_{\text {corr }}^{\text {obse }}$ is the time at the intersection of observation curve with the zero-tide line after phase lag correction, and $t_{\text {prac }}^{\text {the }}$ is the time arriving to the zero-tide point for the practical Newtonian formula of solar tide force, so the left side of the eq. (2) is the time difference between the corrected observation curve and theoretical curve; on the right side of the eq. (2), the first term is time interval during which the gravity travels from the Sun to the ground station by real speed $u$, the second term is time interval during which gravity travels from the Sun to the ground station by the theoretical speed, the speed of light $c$, so the right side is also the time difference between travelling via the actual speed and the theoretical speed derived from the practical formula. If the observation data and data correction are correct, then both sides of eq. (2) have the same physical meaning, and they should equal each other $[10,11]$. If we write $\delta=t_{\text {corr }}^{\text {obse }}-t_{\text {prac }}^{\text {theo }}, u=\alpha c$ and $\varepsilon=\frac{r_{*}}{c}$, eq. (2) becomes

$$
\delta=\frac{\varepsilon}{\alpha}-\varepsilon
$$

Then we have

$$
\alpha=\frac{\varepsilon}{\varepsilon+\delta}
$$

\section{Methods and preliminary results}

We have chosen observational data recorded by the PET gravimeters with rubidium-clocks from the Shiquanhe (abbreviated as SQH) station in Tibet and the Wushi (abbreviated as WS) station in Xinjiang. The two stations are both located inside the western border of China, and are very far away from the Pacific, Indian, Arctic and Atlantic oceans, so the influences of ocean tides to the Earth tide at these two stations are very weak [10-12].

First, we filtered out high frequency disturbances using a low-pass filter with the cutoff frequency of $0.01 \mathrm{~Hz}$; secondly, we applied a phase lag correction of $37 \mathrm{~s}$, which is 
averaged from $38 \mathrm{~s}$ [13], $48 \mathrm{~s}$ [14] of Ray et al. and $25 \mathrm{~s}$ of Arabelos [15]; thirdly, we calculated the tidal factor $\gamma$, the amplitude ratio of the corrected observational curve to practical Newtonian formula of the Earth tides (according to J2000, JPL), and to normalize the observational curve, i.e. to reduce the amplitude of the observational curve by dividing the tidal factor $\gamma$; fourthly, we constructed a quasi-solar tide curve by subtracting Newtonian lunar tidal force from above normalized and corrected observational data; fifthly, we compared the arriving times between the red lines to the blue line, to get the time difference $\delta$; finally, we calculated the travel time $\varepsilon$ of light from the Sun to the Earth, and calculated the speed ratios of gravity to light $\alpha$ according to the eq. (3).

Given that the influence of ocean tide on tidal observations in the deep inland areas of China is very weak, after filtering, drift and relaxation corrections, we are able to compare the observation curves to the curves of the practical Newtonian formula. We have found that there are large phase differences between the observation curve and the curve of the classical Newtonian formula. In contrast, there exists very little phase differences between the observation curve and the curve of the practical Newtonian formula. Now we have a preliminary conclusion that the concept of instant action at a distance seems to be flawed, and the practical formula is probably correct.

Further, we solve the propagation eq. (3) with the observational data of July 7, July 22 and August 5 of 2009 from $\mathrm{SQH}$ station and WS station. After the drift and phase lag corrections, we have the speed ratios of gravity to light as presented in Table 1.

The figures and related data are shown in the Figures $1-3$.

In Figures 2 and 3 black curves denote the filtered curve after $37 \mathrm{~s}$ phase lag correction; red curves denote the fitted curve of the black curves; blue lines denote the practical Newtonian curve; green lines denote the classical Newtonian curve; the time differences between the practical Newtonian curve and the classical Newtonian curve indicate the travelling

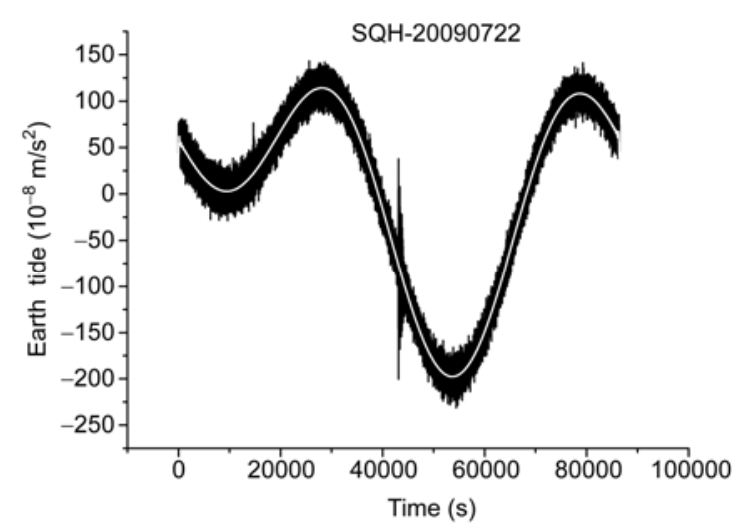

Figure 1 The observational data of SQH station, July 22, 2009. The black curve denotes the original observation curve; the white curve denotes the filtered curve with $0.01 \mathrm{~Hz}$ low pass filter.

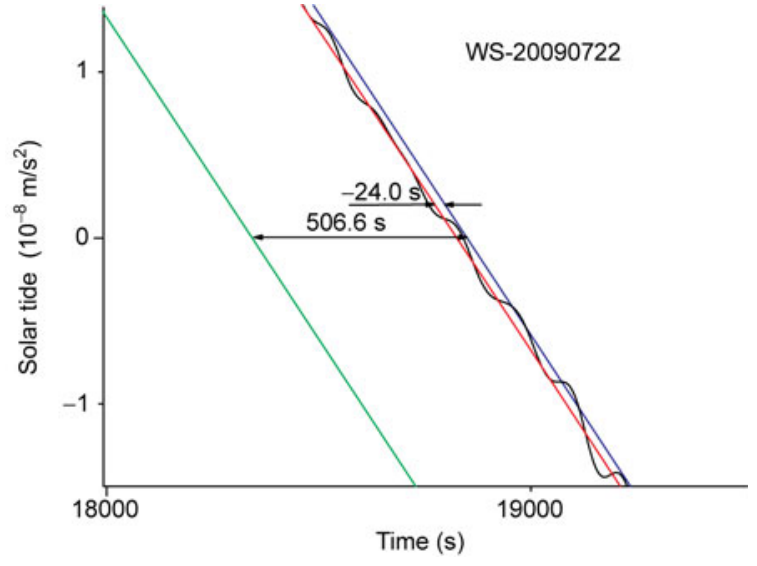

Figure 2 Observational curve, classical and practical Newtonian curves, enhanced near the zero-tide point for WS station, July 22, 2009.

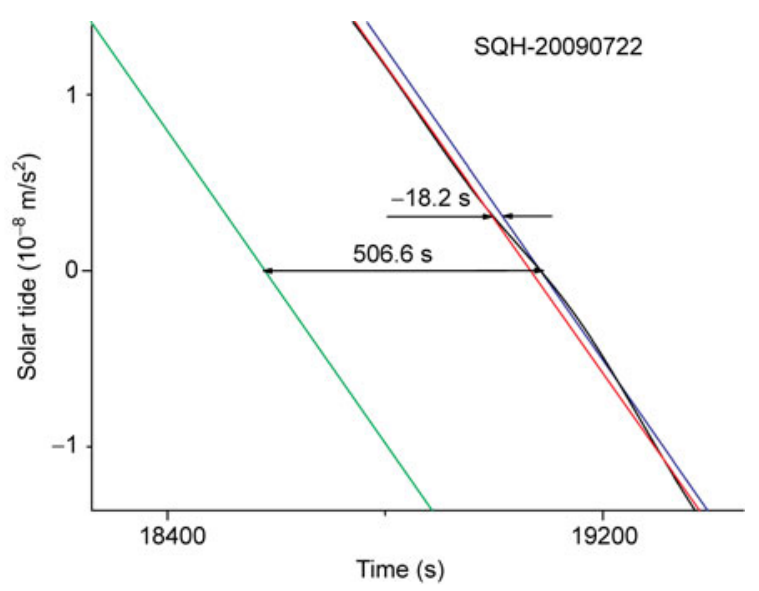

Figure 3 Observational curve, classical and practical Newtonian curves, enhanced near the zero-tide point for SQH station, July 22, 2009.

time of light from the Sun to ground station, i.e. $\varepsilon$ in Table 1; the time differences between the red lines and blue lines indicate the time difference between travelling by the speed of light and by the speed of gravity from the Sun to the ground station, i.e. $\delta$ in Table 1 .

Furthermore, Table 1 shows us the speed ratios of gravity to light, calculated according to the observational data from two stations across three different days.

In Table $1, \varepsilon$ is the time duration during which the light travels from the apparent position of the Sun to the ground station; $\delta$ is the time difference between the corrected observational curve and the practical Newtonian curve; and the speed ratio $\alpha$ of gravity is the ratio of the speed of gravity to the speed of light. The two stations, WS and SQH, are two deep inland stations with little disturbance from the oceans. From Table 1, we can see that the speed ratios of gravity to light are from 0.93 to 1.05 .

Error estimation. The major errors may have three origins:

(1) Observation error, $\Delta \varepsilon$, less than $1 \mathrm{~s}$, could be neglected;

(2) Mean fitting error, $\left|\Delta \delta_{1}\right|$, the time difference between 
Table 1 The travel speeds of the gravity according to the data of Earth tides

\begin{tabular}{|c|c|c|c|c|}
\hline Date & Station & $\varepsilon(\mathrm{s})$ & $\delta(\mathrm{s})$ & $\alpha$ \\
\hline 2009.07 .07 & WS & 507.1 & +5.9 & 0.99 \\
\hline 2009.07 .22 & WS & 506.6 & -24.0 & 1.05 \\
\hline 2009.08 .05 & WS & 505.9 & 36.6 & 0.93 \\
\hline 2009.07 .07 & $\mathrm{SQH}$ & 507.1 & +8.6 & 0.98 \\
\hline 2009.07 .22 & $\mathrm{SQH}$ & 506.6 & -18.2 & 1.04 \\
\hline 2009.08 .05 & $\mathrm{SQH}$ & 505.9 & -14.2 & 1.03 \\
\hline Mean & & 506.5 & -0.9 & $1.00^{+}$ \\
\hline
\end{tabular}

the smoothed curve and real curve, about $5 \mathrm{~s}$;

(3) Phase lag correction error for solar tide $\left|\Delta \delta_{2}\right|$ is about $0.09^{\circ}$ or $21.6 \mathrm{~s}$, according to Ray et al. [13].

We can get the approximate mean relative error of the speed factor of solar gravity as

$$
\begin{gathered}
\alpha=\frac{\varepsilon}{\varepsilon+\delta}, \\
\left|\frac{\Delta \alpha}{\alpha}\right|=\frac{|\Delta \varepsilon||\delta|+|\varepsilon||\Delta \delta|}{|\varepsilon(\varepsilon+\delta)|} \approx \frac{\varepsilon|\Delta \delta|}{|\varepsilon(\varepsilon+\delta)|}, \\
\text { mean value of }\left|\frac{\Delta \alpha}{\alpha}\right| \\
\approx \frac{|\Delta \delta|}{\bar{\varepsilon}+\bar{\delta}}=\frac{\left|\Delta \delta_{1}\right|+\left|\Delta \delta_{2}\right|}{\bar{\varepsilon}+\bar{\delta}} \approx \frac{5.0+21.6}{506.5-0.9} \approx 5 \%,
\end{gathered}
$$

where $\bar{\varepsilon}$ and $\bar{\delta}$ are the mean values of $\varepsilon$ and $\delta$ respectively. From Table 1, we can see that the speed ratios of gravity to light are from 0.93 to 1.05 , with the approximate mean relative error of $5 \%$.

\section{Conclusion and discussion}

From the Earth tide data of WS and SQH stations, we found the first set of observational evidences to show that gravity travels at the speed of light. The reason why we chose the three days shown in Table 1 is because during those days the phases of the moon are either 'new moon' or 'full moon'; in those days the Sun, the Earth and the Moon are almost collinear, and therefore the geometry between the Sun, Earth and Moon is much simple and reliable than other days during which the Moon is at other phases. In recent years, $\mathrm{Xu}$ et al. [16] investigated the translational oscillation of the solid inner core of the Earth through gravity data recorded with superconducting gravimeters (SG); especially, they studied the tidal gravity change in Lhasa with SG measurements [17]. Obviously, the tidal gravity data from SG are more stable and accurate than those from the spring gravimeters; if using SG data in Lhasa, we may find the speed of gravity more accurately. Furthermore, if we have more abundant data of Earth tide from those stations, we may be able to deduce the specific phase lags and the cor- rection for each station.

The authors express great thanks to Pan HuaiWen and Li ZhengYuan of the Network Center of China Earthquake Administration, for kindly providing the Earth tide data of WS and SQH stations, great thanks to Chi Yi, Huang XuChun, Liu JianMing, Liu DuanFa and Lu HongYan, for their technical and other supports, and great thanks to Xi QinWen for beneficial discussion. The authors express great thanks to Li TiPei and Teng JiWen for evaluating and recommending this paper.

1 Einstein A. The Meaning of Relativity. 5th ed. Princeton, NJ: Princeton University Press, 1954

2 Hartle J B. Gravity: An Introduction to Einstein's General Relativity. San Francisco: Addison Wesley, 2008

3 Ohanian H C. Gravitation and Spacetime. New York: W.W. Norton \& Company, Inc. 1976

4 Ohanian H C, Ruffini R. Translated by Xiang S P, Feng L L. Gravitation and Spacetime (in Chinese). Beijing: Science Press, 2006

5 Melchior P. The Tides of Planet Earth. New York: Pergamon Press, 1978

6 Agnew D C. Earth tides. In: Herring T, ed. Treatise on Geophysics. Oxford: Elsevier, 2007. 163-195

7 Tang K Y, Zhang H, Wang Q, et al. Gravity effects of solar eclipse and inducted gravitational field. In: American Geophysical Union, Fall Meeting 2003, abstract \#G32A-0735

8 Tang K Y. The retarded gravitation and paleogravity. In: 27th General Assembly of International Astronomy Union, Rio de Janeiro, Brazil, 2009. 44-45

9 Tang K Y. Total solar eclipse and the pendulum challenging Einstein. Chin Natl Astron, 2009, 11: 40-45

10 Tang K Y. Observational evidences on the speed of gravity (in Chinese). In: Report in the Annul Meeting for the Commission of Gravity and Relativistic Astrophysics of Chinese Society of Physics, Shijiazhuang, China, 2010

11 Tang K Y. Observational evidences to show that gravitational field travels at the speed of light. In: 2010 International Workshop on Gravitational Waves Detection with Atom Interferometer, Hangzhou, China, 2010

12 Tang K Y, Wen W, Hua C C, et al. Observational evidences for the propagation speed of gravity. In: 28th General Assembly of International Astronomy Union, Beijing, China, 2012. 938-939

13 Ray R D, Eanes R J, Chao B F. Detection of tidal dissipation in the solid Earth by satellite tracking and altimetry. Nature, 1996, 381: 595-597

14 Ray R D, Eanes R J, Lemoine F G. Constraints on energy dissipation in the Earth's body tide from satellite tracking and altimetry. Geophys J Int, 2001, 144: 471-480

15 Arabelos D. Comparison of Earth-tide parameters over a large latitude difference. Geophys J Int, 2002, 151: 950-956

16 Xu J Q, Sun H P, Zhou J C. Experimental detection of the inner core translational triplet. Chin Sci Bull, 2010, 55: 276-283

17 Xu J Q, Chen X D, Zhou J C, et al. Characteristics of tidal gravity changes in Lhasa, Tibet, China. Chin Sci Bull, 2012, 57: 2586-2594

Open Access This article is distributed under the terms of the Creative Commons Attribution License which permits any use, distribution, and reproduction in any medium, provided the original author(s) and source are credited. 